\title{
Identification of a new SYT2 variant validates an unusual distal motor neuropathy phenotype
}

Nataly I. Montes-Chinea, MD, Zhuo Guan, PhD, Marcella Coutts, MD, Cecilia Vidal, MD, Steve Courel, BS, Adriana P. Rebelo, PhD, Lisa Abreu, MPH, Stephan Zuchner, MD, PhD, J. Troy Littleton, PhD, and Mario A. Saporta, MD, PhD

Neurol Genet 2018;4:e282. doi:10.1212/NXG.0000000000000282

\section{Abstract \\ Objective}

To report a new SYT2 missense mutation causing distal hereditary motor neuropathy and presynaptic neuromuscular junction (NMJ) transmission dysfunction.

\section{Methods}

We report a multigenerational family with a new missense mutation, c. 1112T >A (p. Ile371Lys), in the C2B domain of SYT2, describe the clinical and electrophysiologic phenotype associated with this variant, and validate its pathogenicity in a Drosophila model.

\section{Results}

Both proband and her mother present a similar clinical phenotype characterized by a slowly progressive, predominantly motor neuropathy and clear evidence of presynaptic NMJ dysfunction on nerve conduction studies. Validation of this new variant was accomplished by characterization of the mutation homologous to the human c. $1112 \mathrm{~T}>\mathrm{A}$ variant in Drosophila, confirming its dominant-negative effect on neurotransmitter release.

\section{Conclusions}

This report provides further confirmation of the role of SYT2 in human disease and corroborates the resultant unique clinical phenotype consistent with heriditary distal motor neuropathy. SYT2-related motor neuropathy is a rare disease but should be suspected in patients presenting with a combination of presynaptic NMJ dysfunction (resembling Lambert-Eaton myasthenic syndrome) and a predominantly motor neuropathy, especially in the context of a positive family history.
Correspondence

Dr. Saporta

mas638@med.miami.edu 


\section{Glossary}

ADM = abductor digiti minimi; AH = abductor hallucis; ANOVA = analysis of variance; APB = abductor pollicis brevis; $\mathbf{C M T}=$ Charcot-Marie-Tooth; CMTNS = Charcot-Marie-Tooth Neuropathy Score; dHMN = Hereditary distal motor neuropathy; eEJC = excitatory evoked junctional current; NMJ = neuromuscular junction; RNS = repetitive nerve stimulation; WT = wildtype.

Hereditary distal motor neuropathies (dHMNs) are a group of rare diseases that share the common feature of a lengthdependent predominantly motor neuropathy. ${ }^{1,2}$ A particular type of dHMN associated with presynaptic neuromuscular junction (NMJ) dysfunction has been recently reported in 2 families harboring autosomal dominant pathogenic mutations in synaptotagmin 2 (SYT2) ${ }^{3,4}$ Here, we describe a new variant in SYT2 causing this same phenotype in a multigenerational family and present functional validation of a dominant-negative effect of the mutation on synaptic transmission in a Drosophila model.

\section{Methods}

\section{Standard protocol approvals, registrations, and patient consents}

Research-related activities were only performed after informed consent was obtained, as part of a University of Miami Institutional Review Board-approved project.

\section{Clinical evaluation}

The proband was evaluated at the Charcot-Marie-Tooth clinic at the University of Miami. Her initial assessment included a full history and physical examination. Disease severity was assessed using the Charcot-Marie-Tooth Neuropathy Score (CMTNS) version $2 .{ }^{5}$ Subsequent evaluation included electrophysiologic studies performed on a Cadwell Sierra Wave EMG machine, following standard protocols. Exercise facilitation was examined by performing a 10-second sustained contraction that was applied to the right abductor pollicis brevis (APB), abductor digiti minimi (ADM), and abductor hallucis (AH) muscles followed by stimulation over their respective motor nerves. Repetitive nerve stimulation (RNS) was performed on the right ulnar nerve recording at the ADM muscle. Ten supramaximal stimuli were applied at $3 \mathrm{~Hz}$, with percentage increment or decrement calculated between the first and fourth response.

\section{Amplification of SYT2 exons and Sanger sequencing}

SYT2 sequence was obtained from University of California Santa Cruz genome browser to design amplification primers. Primers flanking each SYT2 exon were designed using Primer3 software. SYT2 exons were amplified from the proband's and her mother's genomic DNA by PCR with Platinum Taq polymerase (ThermoFisher). Amplifications were carried out in a thermal cycler (Applied Biosystem). PCR products were purified using Qiagen PCR purification kit. Each purified PCR product, corresponding to an exon, was submitted with corresponding sequencing primers to Eurofins for Sanger sequencing. Sequence traces were analyzed using Sequencher (Gene Codes, Ann Arbor, MI).
Following are the primers used for PCR and Sanger sequencing: SYT2-Ex1F: CTTGGTCTCCTCCCCTCACT; SYT2-Ex1R: CCAACCCTACTCACCTCTCG; SYT2-Ex2F: GGCTGACTGTGTACTAATTGGATG; SYT2-Ex2R: CCC AGCCTGAAATCTAAGCA; SYT2-Ex3F: CTCACCCA TTTTTCCCAATG; SYT2-Ex3R: TTAAGGAGGGGAG CAGGTTT; SYT2-Ex4F: GTTCCCACCACACACAGC TC; SYT2-Ex4R: GAGCTATAGGCCCTGCAGTTT; SYT2-Ex5F: CATTTCCCTGCCCCAACT; SYT2-Ex5R: GCCATTGTTCCAGGCTGAG; SYT2-Ex6F: TTTGTC TGTCTCGGCACACT; SYT2-Ex6R: AGGTCGTCTG CCTCCAAAG; SYT2-Ex7F: ACCTTCTCGGCCATCA CATA; SYT2-Ex7R: GGCAGCAAAGTGTTCCTCTT; SYT2-Ex8F: TGGTCTCAGCGGAGTGAAG; SYT2-Ex8R: ACCCAGGCACCATTAGACCT; SYT2-Ex9F: TGGAGCAGAGATGAAACCAA; SYT2-Ex9R: CAGAGCCAGGCTTC TCTTTC.

\section{Genetic screen and Drosophila stocks}

Drosophila melanogaster was cultured on standard medium at $22^{\circ} \mathrm{C}$. Transgenic strains were generated using standard microinjection into white $\left(w^{-/-}\right)$embryos performed by BestGene Inc. UAS-syt1 transgenes were expressed using a GAL4 driver under the control of the pan-neuronal elav promoter, as previously described. ${ }^{6}$ DNA for rescue with individual point mutants was generated using the QuikChange multisitedirected mutagenesis kit (Stratagene, Santa Clare, CA) with the following primer sets: I426K-5'oligo: GGCACCTCCG AACCCAaaGGCCGCTGCATACTTG and I426K-3'oligo: CAAGTATGCAGCGGCCttTGGGTTCGGAGGTGCC.

Wild-type and mutant complementary DNAs were subcloned into a modified pValum construct with an $\mathrm{N}$-terminal myc tag to allow tracking of protein localization in overexpressed animals containing endogenous synaptotagmin 1 (SYT1). These constructs were injected into a yv; attP third chromosome docking strain by BestGene Inc. (Chino Hills, CA). All constructs allowed use of the Gal4/UAS expression system to express the transgenic proteins. UAS-Syt1 transgenes were expressed using a GAL4 driver under the control of the pan-neuronal C155 elav promoter in either control white or syt1 null $\left(\right.$ syt $\left.^{-/-}\right)$backgrounds. Null mutants lacking endogenous SYT1 were generated by crossing Syt1N13, an intragenic Syt1 deficiency, ${ }^{7}$ with Syt1AD4, which truncates SYT1 before the transmembrane domain. ${ }^{8}$

\section{Western blot analysis}

\section{and immunocytochemistry}

Western blotting of whole adult head lysates (1 head/lane) was performed using standard laboratory procedures with 
anti-SYT1 $(1: 1,000$, kindly provided by Noreen Reist) or antisyntaxin (1:1,000, Developmental Studies Hybridoma Bank, Iowa City, IA). Visualization and quantification were done using a LI-COR Odyssey Imaging System (LI-COR Biosciences, Lincoln, MA). Immunostaining was performed on 3rd instar larvae at wandering stage larvae as described previously. ${ }^{9}$ Rabbit myc antibody (1:1,000; Genetex) and anti-horseradish peroxidase (1:1,000; Jackson ImmunoResearch, West Grove, PA) were used for immunostaining. Confocal stacks of muscle 6 and 7 NMJs containing immunoreactive proteins were captured on a Zeiss Pascal Confocal with PASCAL software (Carl Zeiss MicroImaging, Inc.) using a $63 \times$ numerical aperture 1.3 Plan Neofluar oil immersion lens (Carl Zeiss, Inc) and fluorescent secondary antibodies (Molecular Probes, Carlsbad, CA).

\section{Electrophysiology}

Postsynaptic currents from third instar male larvae at the wandering stage from the indicated genotypes were recorded at muscle fiber 6 of segment A3 using 2-electrode voltage clamp with a $-80 \mathrm{mV}$ holding potential in hemolymph-like (HL) 3.1 saline solution as previously described. ${ }^{6,10}$ Final calcium concentration was adjusted to $2 \mathrm{mM}$. For evoked and mini analysis, $\mathrm{n}$ refers to the number of NMJs analyzed, with no more than 2 NMJs analyzed per animal, and with animals derived from at least 3 independent experiments. Data acquisition and analysis was performed using Axoscope 9.0 and Clampfit 9.0 software (Molecular Devices, Sunnyvale, CA). Motor nerves innervating the musculature were severed and placed into a suction electrode, so action potential stimulation could be applied at the indicated frequencies using a programmable stimulator (Master8; AMPI, Jerusalem, Israel).

\section{Data analysis and statistics}

Electrophysiology analysis was performed using Clampfit 10 software (Axon Instruments, Foster City, CA), as previously described. ${ }^{6}$ Statistical analysis and graphs were performed using Origin Software (OriginLab Corporation, Northampton, MA). Statistical significance was determined using 1-way analysis of variance (ANOVA) (nonparametric) with post hoc Sidak multiple comparisons test. The $p$ values associated with 1-way ANOVA tests were adjusted $p$ values obtained from a post hoc Sidak multiple comparisons test. Appropriate sample size was determined using GraphPad Statmate. In all figures, the data are presented as mean \pm SEM. Statistical comparisons are with control, unless noted. The results were all shown: N.S. $=$ no significant change $(p>0.05),{ }^{*} p<0.05,{ }^{* *} p<0.005,{ }^{* * *} p<$ 0.001 , and ${ }^{* * *} p<0.0001$. All error bars are SEM.

\section{Data availability}

Data, methods, and materials used to conduct this research are documented in detail in the Methods section.

\section{Results}

\section{Clinical evaluation}

The Proband is a 50-year-old woman who was referred to the University of Miami Comprehensive CMT clinic for evaluation of suspected CMT because of gradually progressive weakness of her extremities. She had normal developmental milestones but was found to have bilateral high arched feet and hammertoes and occasional falls around the age of 8 years. She developed progressive leg weakness, worsening bilateral hand cramping, weak handgrip, and only mild paresthesia on distal extremities. The proband's family history is remarkable for similar symptoms reported by her mother, maternal grandfather, 2 maternal uncles, 1 maternal aunt, a younger sister, and a nephew (figure 1A). Initial physical examination revealed bilateral pes cavus and hammer toes (figure 1B), inability to walk on heels or toes, severe, nonfatigable, distal lower extremity weakness, limited range of motion on ankles bilaterally because of ankle fusions, nonspecific sensory changes in lower extremities, and diffuse hyporreflexia with postexercise normalization. Her initial CMTNS was 9. Her mother, a 68-year-old woman, was also evaluated. She has had a history of high-arched feet and hammertoes since childhood. Examination showed bilateral pes cavus and hammer toes (figure 1B). Strength testing revealed intrinsic hand muscle and plantarflexion weakness. Deep tendon reflexes were absent initially, with postexercise facilitation noted. Sensory examination was essentially normal for touch, pinprick, vibration, and proprioception. Her CMTNS was 4 .

\section{Clinical neurophysiology testing}

The proband underwent electrodiagnostic testing to further evaluate for a hereditary peripheral neuropathy. Sensory nerve conduction studies were normal. However, compound muscle action potential amplitudes were significantly reduced throughout all tested motor nerves. Examination of each motor nerve incidentally showed decreasing amplitudes with successive stimulations. For this reason, a 10-second sustained contraction was applied at the right abductor digiti minimi $(\mathrm{ADM}), \mathrm{APB}$, tibialis anterior, and $\mathrm{AH}$, and their respective motor nerves were stimulated afterward. This resulted in significant incremental responses (figure 1C). RNS was subsequently performed on the right ulnar nerve recording at the right $\mathrm{ADM}$ and revealed a $42 \%$ decremental response and a $125 \%$ postexercise facilitation (figure $1, \mathrm{C}$ and $\mathrm{D}$ ). Needle EMG examination revealed evidence of ongoing denervation in the form of positive waves and fibrillation potentials and of chronic reinnervation in the form of reduced recruitment in the medial gastrocnemius muscles. The proband's mother also underwent a limited nerve conduction studies and RNS examination with similar findings, including normal sural sensory response, $17.6 \%$ decremental response and an $86 \%$ postexercise facilitation on repetitive stimulation study (figure 1C). To rule out paraneoplastic Lambert-Eaton syndrome, the proband was investigated further and antibodies against voltage-gated calcium channel were negative, and a chest $\mathrm{CT}$ did not show evidence of lung carcinoma.

\section{Genetic analysis}

Sanger sequencing of the SYT2 gene revealed a heterozygous $1112 \mathrm{~T}>\mathrm{A}$ missense mutation in both the proband and her mother (figure $1 \mathrm{E}$ ). This mutation causes an isoleucine (I) to 


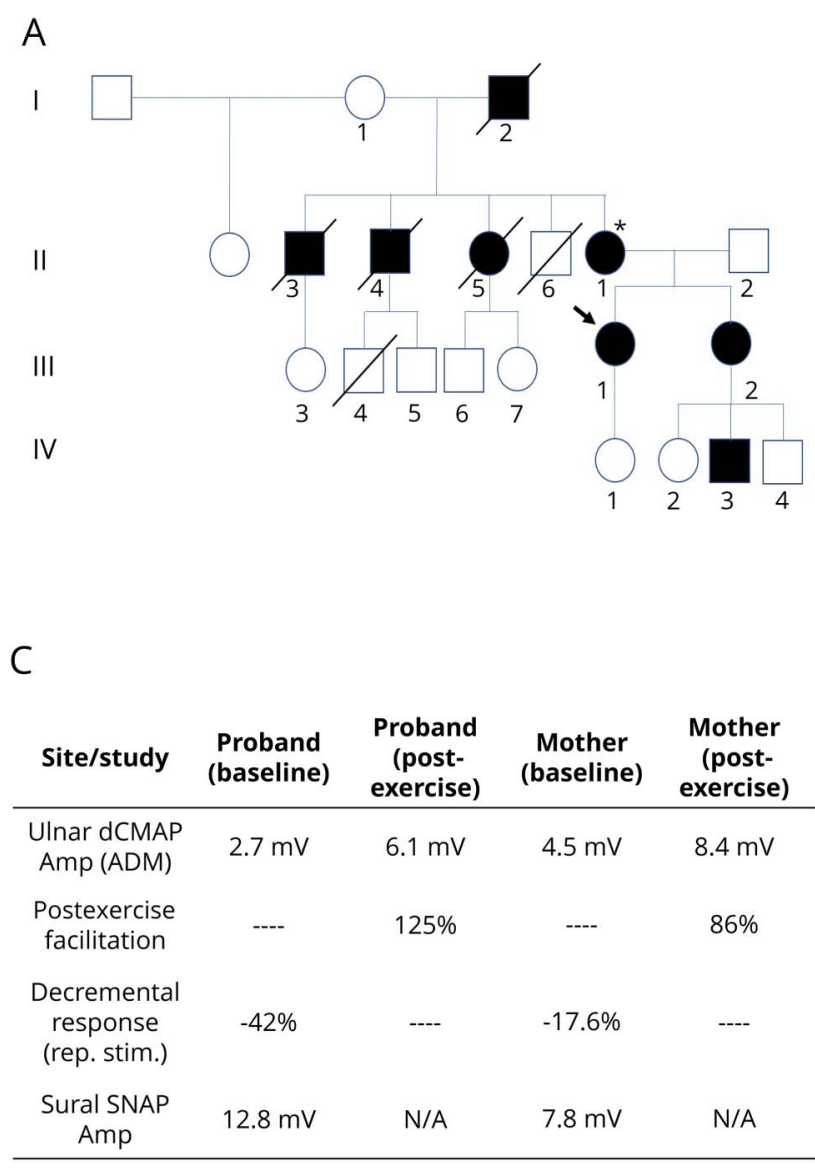

$\mathrm{E}$

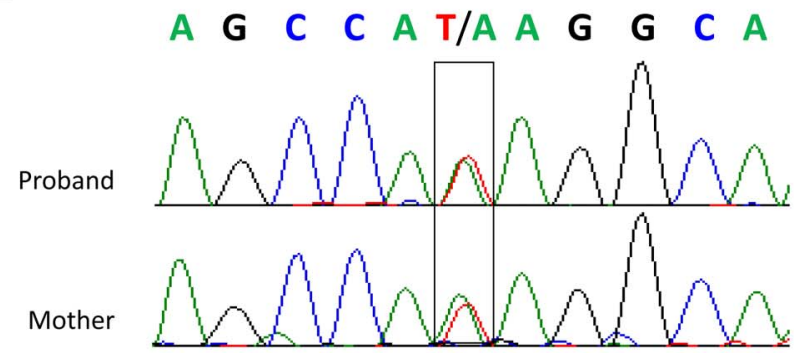

1112 T>A AACGAAGCCAAAGGCAAGATC
B

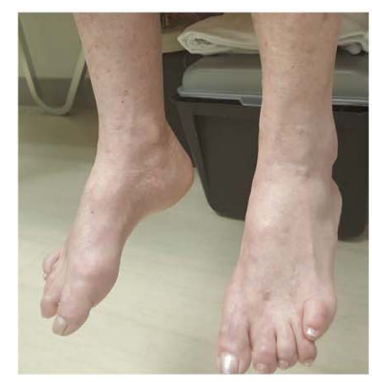

III-1: Proband

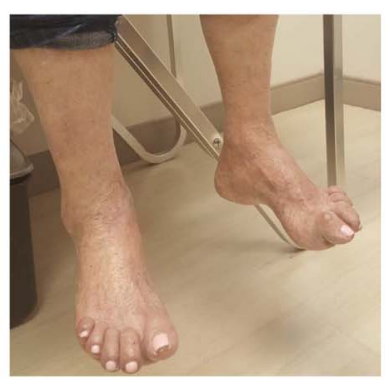

II-1: Mother
D
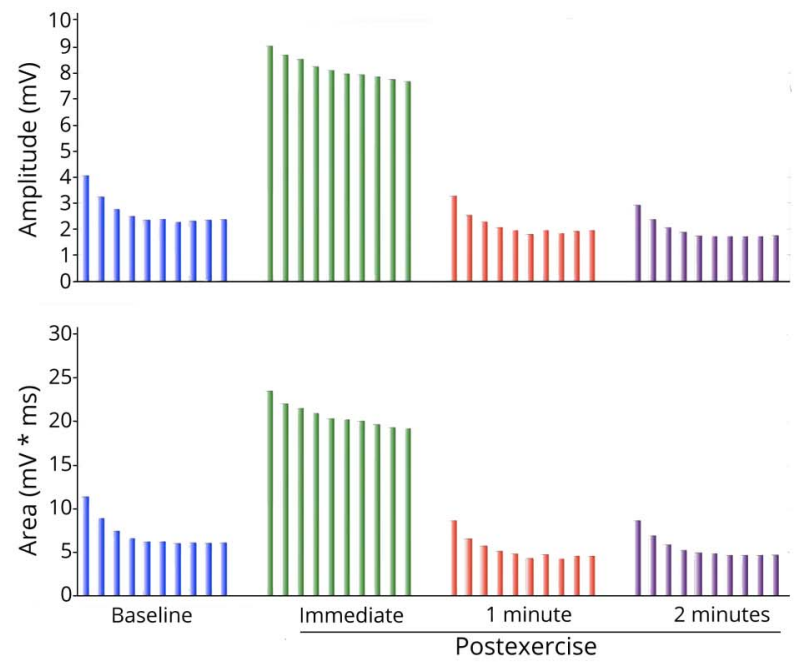

$\mathrm{F}$

Human D Y D K L G K N E A I G K I F V G S N A T G Human (1371k) D Y D K L G K N E A K G K I F V G S N A T G P. troglodytes $D$ Y $D$ K L L G K K N E A I G $K$ K I F F V G S N N A T G M. mulatta D Y D K L GKN E A I G K I F V G S N A T G Fcatus D $\quad$ Y $D$ D K L G K K N E A I G K I F V G S S N A T G M. musculus D Y D K L G K N E A I G K I F V G S N A T G D. rerio D $\quad$ Y $D$ D $K$ I G K K N D A I G K I F I G S K A S G D. melanogaster $D$ Y $D$ R I G T S E P I G R C I L G C M G T G C. elegans D Y D K L G S N D A I GR $R$ C L L G C N G T G X. tropicalis D Y D K L G K NE A I G K I F V G C N A S G

(A) Family pedigree reveals multiple affected family members supporting autosomal dominant inheritance. Filled symbols are clinically affected individuals, arrow denotes the proband. (B) Both the proband and her mother presented long-standing pes cavus and hammer toes. (C) Table summarizing electrophysiologic findings for the proband and her mother. Main findings were significant postexercise facilitation in compound muscle action potential amplitudes, significant decremental response to $3 \mathrm{~Hz}$ repetitive stimulation, and normal sural sensory responses. (D) Graph summary of the proband's repetitive stimulation study demonstrating both decremental response to $3 \mathrm{~Hz}$ repetitive stimulation and significant postexercise increment. (E) Sanger sequencing revealed a 1112T>A substitution in both the proband and her mother, resulting in an isoleucine (I) to lysine (K) change at residue 371 . (F) This residue is highly conserved across species. $\mathrm{ADM}=$ abductor digiti minimi; amp = Amplitude; $\mathrm{dCMAP}=$ distal compound muscle action potential; N/A = not applicable; Rep. stim. = repetitive nerve stimulation; SNAP = sensory nerve action potential. 
lysine (K) change at residue 371 (I371K). The I371K residue is located within the C2B domain of SYT2, which spans residues 271 to 406 . This variant was not found in the Genome Aggregation Database (gnomAD). Further variant analysis using the PROVEAN (-6.243), SIFT (0.0), PolyPhen-2 (1.000), and Align-GVGD (Grantham variation [GV]: 0, Grantham deviation [GD]: 101.61, class: C65) scores predicted this variant to be disease causing. PhyloP $(+4.83)$ and PhastCons (0.99) scores showed this variant to be highly conserved throughout different species (figure $1 \mathrm{~F}$ ).

\section{Functional validation of the new I371K SYT2 variant in Drosophila}

To examine how the I371K mutation affects synaptic transmission directly, we generated transgenic Drosophila expressing the mutant gene using the GAL4-UAS expression system. Drosophila has a single homolog (DSYT1) of the mammalian synaptic vesicle SYT subfamily that includes SYT1, SYT2, and SYT9. DSYT1 and human SYT2 share strong homology with conservation of the key residues that form the $\mathrm{C} 2 \mathrm{~B}$ calcium binding pocket, including I371 (corresponding to I426 in Drosophila) and 2 previous $\mathrm{C} 2 \mathrm{~B}$ residues that were found mutated in patients (D307 and P308, figure 2A). We generated wildtype (WT) and I371K UAS-DSyt1 transgenic lines and expressed the transgenes in $s y t 1^{-/-}$null mutants with the panneuronal GAL4 driver elav ${ }^{C 155}$. Drosophila Syt ${ }^{-/-}$null mutants show a reduction in viability during larval development $(50 \%$ survival to the 3rd instar stage) because of defective synaptic transmission. In contrast to the ability of WT SYT1 to rescue lethality (100\% survival), SYT1 I426K not only failed to rescue but also caused a dramatic reduction in viability $(2.9 \%)$ compared to null mutants alone (50\%) (figure 2B). Western blot analysis indicated that the I426K SYT 1 protein was expressed at similar levels to WT (figure 2C). Immunocytochemistry demonstrated that the protein also localized normally at NMJ synapses (figure $2 \mathrm{D}$ ), suggesting the dominant effects on viability are secondary to aberrant function of SYT1 I426K versus degradation or abnormal localization. To analyze neurotransmitter release in Syt1 nulls that were rescued with either WT or I426K SYT1, we performed current recordings of postsynaptic responses in voltage-clamp at 3rd instar NMJs. As observed in the behavioral viability assays, I426K rescued animals displayed synaptic transmission defects that were worse than the Syt1 null mutant. I426K synapses displayed severely defective synchronous neurotransmitter release (figure 2, E-G), a large increase in failure rate following stimulation (figure $2 \mathrm{H}$ ) and enhancement of the slower asynchronous phase of release (figure 2I). I426K also failed to rescue the elevated rate of spontaneous fusion observed in Syt1 null mutants. These results indicate that the SYT1 I426K mutation eliminates the ability of the protein to drive calcium-triggered neurotransmitter release and acts dominantly to reduce the residual release that is normally observed in the absence of SYT1.

To model the dominant phenotype observed in patients that have one endogenous copy of WT SYT2, we characterized the effects of overexpressing I426K on synaptic transmission in the presence of endogenous DSYT1. Two independent transgenic insertions (I426K\#1 and 1426K\#2) were overexpressed using the elav ${ }^{\mathrm{C} 155}$ driver. Compared to overexpression of WT SYT1, both I426K lines resulted in a strong dominant-negative effect on action potential evoked release, decreasing the excitatory evoked junctional current (eEJC) by $68.5 \%$ and $64.8 \%$, respectively (figure $3, \mathrm{~A}$ and $\mathrm{B}$ ). In addition, both $\mathrm{I} 426 \mathrm{~K}$ transgenic overexpression lines showed facilitation of the eEJC during $10 \mathrm{~Hz}$ stimulation compared to the synaptic depression observed in controls (figure 3, C and D), consistent with a reduction in the initial release probability caused by SYT1 I426K. These findings indicate that SYT I426K lacks normal function for promoting synaptic vesicle fusion and exerts a strong dominant-negative effect on synaptic transmission even in the presence of WT SYT1.

\section{Discussion}

Gain-of-function mutations in SYT2 have been recently associated with an autosomal dominant presynaptic congenital myasthenic syndrome in 2 families. SYT2 is an integral membrane protein of synaptic vesicles and serves as a calcium sensor for neurotransmitter release, with calcium binding to its $\mathrm{C} 2 \mathrm{~B}$ domain activating vesicle fusion. ${ }^{11-13}$ Of interest, the clinical presentation varied between families and family members, ranging from a presynaptic myasthenic disorder resembling LambertEaton syndrome and a distal motor neuropathy, or even a combination of both. Here, we studied a multigenerational family with a new SYT2 mutation presenting with clear evidence of both presynaptic neuromuscular transmission impairment as well as a distal motor neuropathy, both clinically and electrophysiologically. This report therefore reinforces the link between SYT2 mutations and distal hereditary motor neuropathy.

Synaptotagmins 1 and 2 have been shown to have several major roles in regulating synaptic vesicle fusion. Their major function is as calcium sensors for driving fast synchronous fusion of synaptic vesicles at synapses. However, they also function to suppress the slower asynchronous fusion pathway, ensuring that release is tightly linked to the action potential. As such, in the absence of synaptotagmin 1 , increased asynchronous release is observed because of loss of this suppression function. The I426K mutant disrupts the normal function of synaptotagmin 1, including its role in driving fast synchronous fusion, as well as its role in suppressing asynchronous release.

Synaptotagmin 1 null mutants die throughout development, with many dying during the larval stage. However, when placed directly on food where little movement is required, the animals can occasionally survive to adulthood, although they show severe motor defects. Similar to other species, including mammals, synaptotagmin 1 mutants still have residual slow neurotransmitter release. Although the identity of the residual "asynchronous" calcium sensor is still being debated, recent studies suggest that another member of the synaptotagmin family-synaptotagmin 7 -may play this role. ${ }^{14,15}$ This explains the 50\% survival observed in SYT null mutants. 
Figure 2 I426K DSYT1 fails to rescue neurotransmitter release in synaptotagmin null mutants
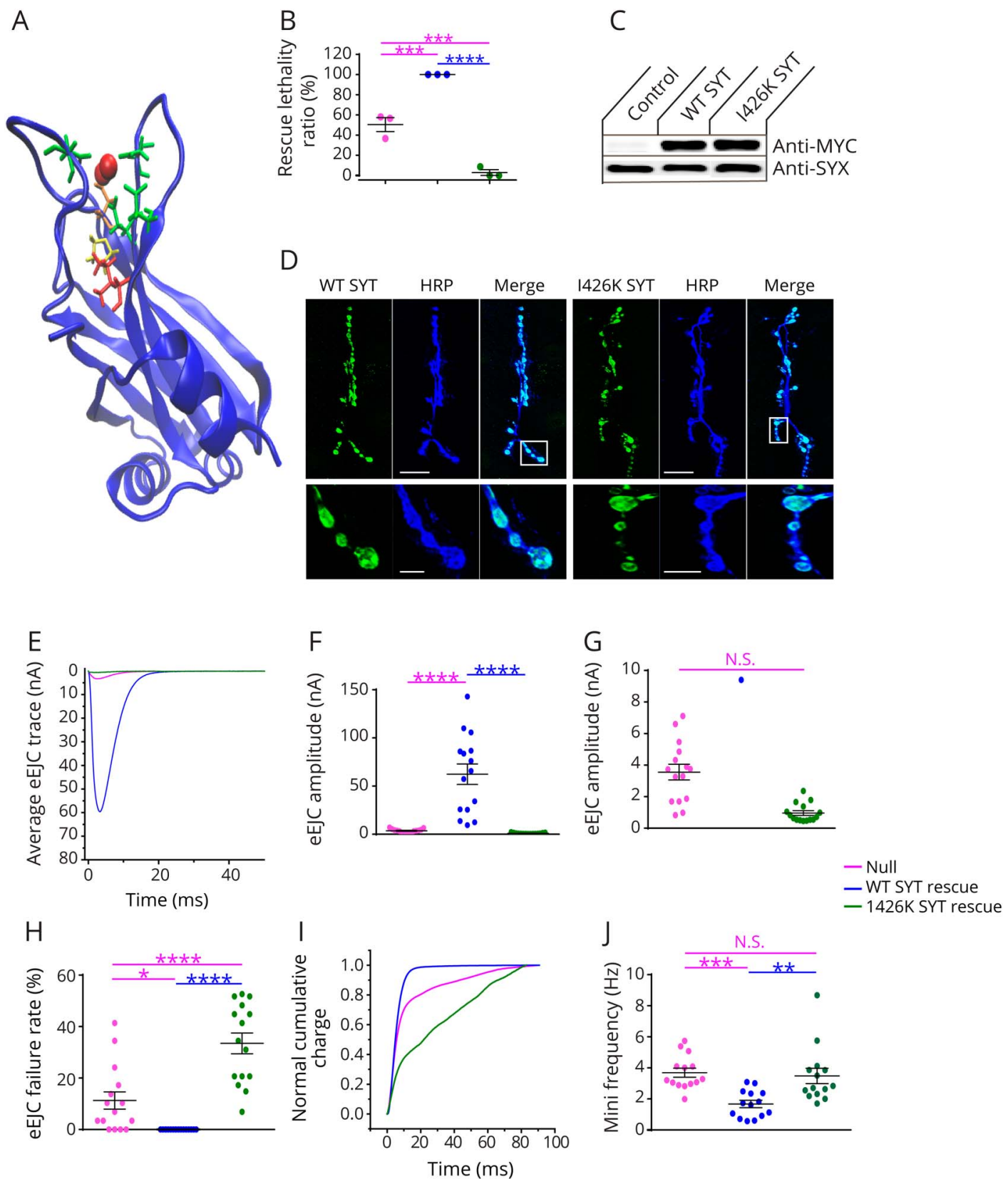

(A) Stereoview of residues found to be mutated in human synaptotagmin 2 patients (Asp307 [orange], Pro308 [yellow], and lle371 [red]) modeled on the rodent synaptotagmin 1 C2B crystal structure. The 5 essential calcium binding residues are highlighted in green and calcium ions in red. (B) The ability of transgenes to rescue the lethality of Syt1 null mutants are shown. Larvae were collected at the 1 st instar larval stage and placed into food vials. The number of animals surviving to the $3 \mathrm{rd}$ instar wandering larval stage was quantified. Quantification of average rescue ratio (\%) for the indicated genotypes (Syt $1^{-1-}$ null, $50.5 \pm 6.9, \mathrm{n}=3$; elav $^{\mathrm{C} 155}$-GAL4; Syt1 ${ }^{-1-}$; UAS-SYT1, $100 \pm 0, \mathrm{n}=3$; elav ${ }^{\mathrm{C} 155}$-GAL4; Syt1 1/-; UAS-SYT1 $1426 \mathrm{~K}, 2.9 \pm 2.9, \mathrm{n}=3$ ). (C) Western blot with anti-MYC (top panel) or anti-syntaxin (SYX-control) antisera from head extracts of control or transgenic Drosophila: WT (control), WT DSYT or I426K DSYT induction by elav $^{\mathrm{C} 155}$-GAL4. (D) Representative larval NMJs stained with anti-MYC antisera (green) for animals with MYC-tagged WT or 1426K SYT1. The axon is stained with the neuronal marker anti-HRP (blue). The boxed area is magnified in the bottom panel. Mutant SYT1 targets normally to presynaptic terminals. Scale bar-20 $\mu \mathrm{M}$-top panels: $5 \mu \mathrm{M}$-bottom panels. (E) Representative eEJCs recorded in $2 \mathrm{mM}$ external calcium in Syt $1^{-1-}$ null larvae (red) and null mutants rescued with WT DSYT1 (blue) or 1426K DSYT1 (green). (F) Quantification of mean eEJC amplitudes in the indicated genotypes: Syt1 ${ }^{-1-}$ null, $3.6 \pm 0.5 \mathrm{nA}, \mathrm{n}=15$; elav 155 -GAL4; Syt1 $1^{-1-}$; UAS-SYT1, $62.3 \pm 10.6 \mathrm{nA}, \mathrm{n}=15$; elav ${ }^{\mathrm{C} 155}$-GAL4; Syt $1^{-1 /}$; UAS-SYT1 I426K, $1.0 \pm 0.2 \mathrm{nA}, \mathrm{n}=15$. (G) Expanded vertical axis scale of the data shown in (F). (H) Failure rates (\%) as calculated by counting trials with no detectable eEJCs within $10 \mathrm{~ms}$ during 30 consecutive stimuli given at $0.5 \mathrm{~Hz}$ for the indicated

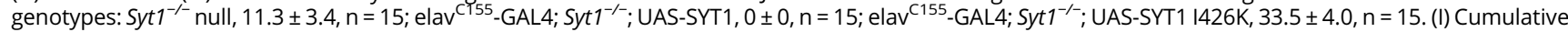
vesicle release defined by charge transfer normalized for the maximum in $2.0 \mathrm{mM}$ calcium for each genotype (same color code as in E). Each trace was adjusted to a double exponential fit. Both the null and $1426 \mathrm{~K}$ rescued animals display a prominent increase in the slow asynchronous phase of release. (J) Quantification of mini frequency in the indicated genotypes: Syt $1^{-/-}$null, $3.7 \pm 0.3 \mathrm{~Hz}, \mathrm{n}=14$; elav ${ }^{\mathrm{C} 155}-\mathrm{GAL} 4 ;$ Syt1 $1^{-1-}$; UAS-SYT1, $1.7 \pm 0.2 \mathrm{~Hz}, \mathrm{n}=14 ;$ elav 155 GAL4; Syt $1^{-1-}$; UAS-SYT1 1426K, $3.5 \pm 0.5 \mathrm{~Hz}, \mathrm{n}=14$. Statistical significance was determined using 1 -way analysis of variance (nonparametric) with post hoc Sidak multiple comparisons test. N.S. $=$ no significant change $(p>0.05)$, $* p<0.05, * \star p<0.005, \star \star \star x p<0.001$, and $* \star \star \star p<0.0001$. All error bars are SEM. eEJC $=$ excitatory junctional current; HRP = horseradish peroxidase; NMJ = neuromuscular junction; $\mathrm{WT}=$ wildtype. 

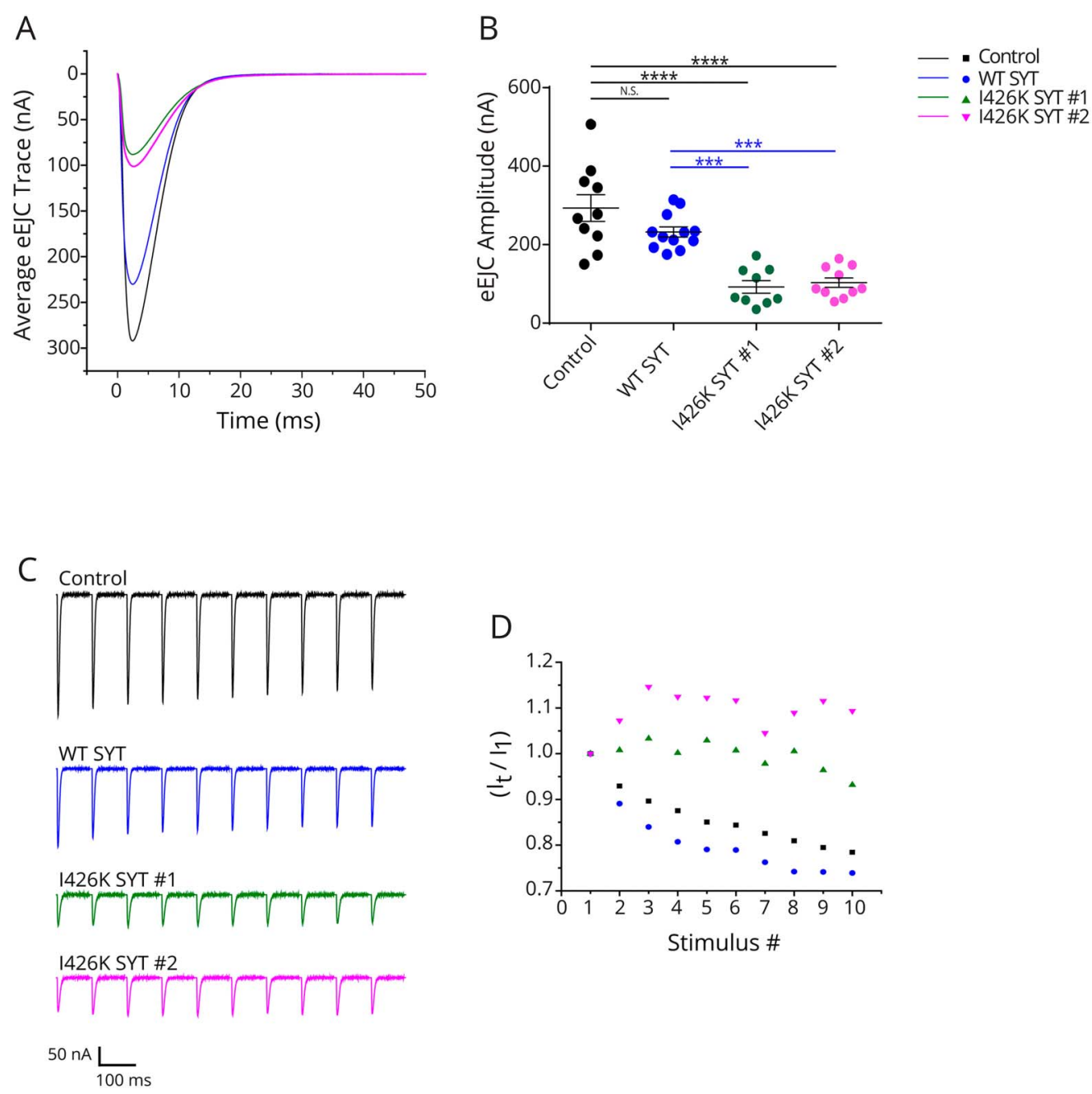

(A) Representative eEJCs recorded in $2 \mathrm{mM}$ extracellular calcium at 3rd instar larval muscle 6 NMJs for the indicated genotypes (control, overexpression of WT or 1426K DSYT1 with elav ${ }^{\mathrm{C} 155}{ }_{\text {GAL }}$ ). (B) Quantification of mean eEJC amplitude in the indicated genotypes: control elav ${ }^{\mathrm{C} 155}$-GAL4, $293.3 \pm 34.2 \mathrm{nA}$, $\mathrm{n}=10$; elav $^{\mathrm{C} 155}$-GAL4; UAS-SYT1, $223.9 \pm 11.7 \mathrm{nA}, \mathrm{n}=12$; elav ${ }^{\mathrm{C} 155}$-GAL4; UAS-SYT1 I426K\#1, $92.4 \pm 15.9 \mathrm{nA}, \mathrm{n}=9$; elav ${ }^{\mathrm{C} 155}$-GAL4; UAS-SYT1 I426K\#2, $103.2 \pm 12.2 \mathrm{nA}, \mathrm{n}=$ 10). (C) Representative eEJCs during a $10 \mathrm{~Hz}$ tetanic nerve stimulation in $2 \mathrm{mM}$ external calcium for the indicated genotypes. (D) The average eEJC for the first 10 responses normalized to the amplitude of the first response during a 10-Hz tetanus is shown for control (black), elav ${ }^{\text {(155 }}$-GAL4; UAS-WT DSYT 1 (blue), elav ${ }^{\mathrm{C15}}$-GAL4; UAS-DSYT1 1426K \#1 (green) and elav ${ }^{\mathrm{C} 155}$-GAL4; and UAS-DSYT1 1426K \#2 (magenta). Statistical significance was determined using 1-way

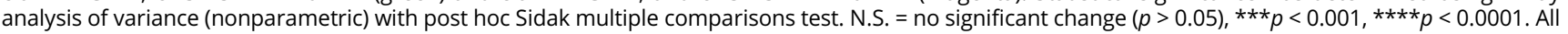
error bars are SEM. eEJC = excitatory junctional current; NMJ = neuromuscular junction.

There is no known effect on localization of WT synaptotagmin 1 when the I426K mutant is co-expressed. Our work and others have indicated that multimerization of synaptotagmin 1 into oligomeric complexes is required for neurotransmitter release. ${ }^{6}$ Our current data indicate that the mutant I426K version forms mixed oligomers with WT synaptotagmin 1, and the loss of normal calcium binding by I426K poisons the multimeric complex that includes WT synaptotagmin, thus dramatically reducing neurotransmitter release and leading to the autosomal dominant phenotypes.

The role of NMJ impairment in the pathophysiology of inherited peripheral neuropathies has been the focus of several recent studies. NMJ transmission dysfunction is now well documented in several types of inherited neuropathies, including Congenital Hypomyelinating Neuropathy (PMP22 point mutations), ${ }^{16} \mathrm{CMT} 1 \mathrm{~B}(\mathrm{MPZ}),{ }^{17} \mathrm{CMT} 2 \mathrm{D}$ (GARS), ${ }^{18-20}$ and CMT2O (DYNC1H1). ${ }^{21}$ Findings include reduced size, branching, and complexity of NMJs by immunostaining studies, altered growth and maturation of the NMJs, reduced amplitudes of nerve-evoked muscle endplate potentials, and NMJ transmission failure during sustained nerve stimulation. In 2 different mouse models of CMT2D (Gars ${ }^{\mathrm{P} 278 \mathrm{KY}}$ and Gars ${ }^{\mathrm{C} 201 \mathrm{R}}$ ), the NMJ deficits correlated with disease severity and progressed with age. ${ }^{20}$ These recent studies demonstrated that dysfunction and degeneration of NMJs are often an early pathologic finding even in primarily demyelinating inherited neuropathies and precede impairments in axonal conduction. Nevertheless, it is likely that, in this context, NMJ impairment is secondary to the distal, length-dependent axonal degeneration 
process characteristic of most types of inherited neuropathies and not directly linked to specific presynaptic transmission dysfunction. Of note, no electrophysiologic evidence of presynaptic NMJ transmission has been previously reported in patients with CMT.

This concept was challenged recently by the identification of mutations in the presynaptic choline transporter SLC5A7 as a cause of distal hereditary motor neuropathy type VII (dHMN VII). ${ }^{22}$ SLC5A7 is a $\mathrm{Na}^{+} / \mathrm{Cl}^{-}$-dependent highaffinity transporter that mediates uptake of choline for acetylcholine synthesis and therefore is a critical determinant of synaptic acetylcholine synthesis and release at the NMJ. Of interest, as is the case for SYT2 mutations, patients with dHMN VII do not present the hallmark clinical features of a congenital myasthenic syndrome, namely, ophthalmoparesis, ptosis, bulbar weakness, and respiratory fatigable weakness. Nonetheless, the role of these 2 presynaptic NMJ proteins in the etiology of hereditary motor neuropathies provides a new framework to investigate the connections between NMJ transmission and axonal biology and function.

The clinical findings in our patients are in keeping with the phenotype described in 2 families with SYT2 mutations, ${ }^{3}$ including foot deformities since childhood, distal weakness, minimal sensory findings, and reduced deep tendon reflexes with evidence of postexercise facilitation. The electrodiagnostic features were also in agreement with the previous report, demonstrating clear presynaptic neuromuscular transmission dysfunction characterized by decremental response to RNS and significant postexercise facilitation and normal sensory responses. We identified a new SYT2 mutation causing autosomal dominant distal hereditary motor neuropathy, confirming the interesting connection between presynaptic neuromuscular transmission dysfunction and motor axonopathy.

\section{Study funding}

No targeted funding reported.

\section{Disclosure}

N.I. Montes-Chinea, Z. Guan, M. Coutts, C. Vidal, S. Courel, A.P. Rebelo, L. Abreu, and S. Zuchner report no disclosures. J. T. Littleton has received research support from NIH. M.A. Saporta has served on scientific advisory boards for the Charcot-Marie-Tooth Association and Acceleron; serves on the editorial board of the Journal of the Peripheral Nervous System; has been a consultant for Alnylam, Strongbridge, Biogen, and Serepta; and has received research support from the Charcot-Marie-Tooth Association. Full disclosure form information provided by the authors is available with the full text of this article at Neurology.org/NG.

Received June 11, 2018. Accepted in final form August 21, 2018.

\section{References}

1. Rossor AM, Kalmar B, Greensmith L, Reilly MM. The distal hereditary motor neuropathies. J Neurol Neurosurg Psychiatry 2012;83:6-14.

2. Bansagi B, Griffin H, Whittaker RG, et al. Genetic heterogeneity of motor neuropathies. Neurology 2017;88:1226-1234.

3. Herrmann DN, Horvath R, Sowden JE, et al. Synaptotagmin 2 mutations cause an autosomal-dominant form of lambert-eaton myasthenic syndrome and nonprogressive motor neuropathy. Am J Hum Genet 2014;95:332-339.

4. Whittaker RG, Herrmann DN, Bansagi B, et al. Electrophysiologic features of SYT2 mutations causing a treatable neuromuscular syndrome. Neurology 2015;85: 1964-1971.

5. Murphy SM, Herrmann DN, McDermott MP, et al. Reliability of the CMT neuropathy score (second version) in Charcot-Marie-Tooth disease. J Peripher Nerv Syst 2011;16:191-198.

6. Guan Z, Bykhovskaia M, Jorquera RA, Sutton RB, Akbergenova Y, Littleton JT. A synaptotagmin suppressor screen indicates SNARE binding controls the timing and Ca2+ cooperativity of vesicle fusion. Elife 2017;6:e28409.

7. Littleton JT, Stern M, Perin M, Bellen HJ. Calcium dependence of neurotransmitter release and rate of spontaneous vesicle fusions are altered in Drosophila synaptotagmin mutants. Proc Natl Acad Sci U S A 1994;91:10888-10892.

8. DiAntonio A, Schwarz TL. The effect on synaptic physiology of synaptotagmin mutations in Drosophila. Neuron 1994;12:909-920.

9. Huntwork S, Littleton JT. A complexin fusion clamp regulates spontaneous neurotransmitter release and synaptic growth. Nat Neurosci 2007;10:1235-1237.

10. Jorquera RA, Huntwork-Rodriguez S, Akbergenova Y, Cho RW, Littleton JT. Complexin controls spontaneous and evoked neurotransmitter release by regulating the timing and properties of synaptotagmin activity. J Neurosci 2012;32:18234-18245.

11. Littleton JT, Stern M, Schulze K, Perin M, Bellen HJ. Mutational analysis of Drosophila synaptotagmin demonstrates its essential role in $\mathrm{Ca}(2+)$-activated neurotransmitter release. Cell 1993;74:1125-1134.

12. Mackler JM, Drummond JA, Loewen CA, Robinson IM, Reist NE. The C(2)B $\mathrm{Ca}(2+)$-binding motif of synaptotagmin is required for synaptic transmission in vivo. Nature 2002;418:340-344.

13. Pang ZP, Melicoff E, Padgett D, et al. Synaptotagmin-2 is essential for survival and contributes to $\mathrm{Ca} 2+$ triggering of neurotransmitter release in central and neuromuscular synapses. J Neurosci 2006;26:13493-13504.

14. Luo F, Südhof TC. Synaptotagmin-7-Mediated asynchronous release Boosts highfidelity synchronous transmission at a central synapse. Neuron 2017;94:826-839.e3.

15. TurecekJ, Regehr WG. Synaptotagmin 7 mediates both facilitation and asynchronous release at granule cell synapses. J Neurosci 2018;38:3240-3251.

16. Scurry AN, Heredia DJ, Feng CY, Gephart GB, Hennig GW, Gould TW. Structural and functional abnormalities of the neuromuscular junction in the trembler-J homozygote mouse model of congenital hypomyelinating neuropathy. J Neuropathol Exp Neurol 2016;75:334-346.

17. Patzkó A, Bai Y, Saporta MA, et al. Curcumin derivatives promote Schwann cell differentiation and improve neuropathy in R98C CMT1B mice. Brain 2012;135: 3551-3566.

18. Sleigh JN, Grice SJ, Burgess RW, Talbot K, Cader MZ. Neuromuscular junction maturation defects precede impaired lower motor neuron connectivity in CharcotMarie-Tooth type 2D mice. Hum Mol Genet 2014;23:2639-2650.

19. Grice SJ, Sleigh JN, Motley WW, et al. Dominant, toxic gain-of-function mutations in gars lead to non-cell autonomous neuropathology. Hum Mol Genet 2015;24: 4397-4406.

20. Spaulding EL, Sleigh JN, Morelli KH, Pinter MJ, Burgess RW, Seburn KL. Synaptic deficits at neuromuscular junctions in two mouse models of Charcot-Marie-Tooth type 2d. J Neurosci 2016;36:3254-3267.

21. Sabblah TT, Nandini S, Ledray AP, et al. A novel mouse model carrying a human cytoplasmic dynein mutation shows motor behavior deficits consistent with CharcotMarie-Tooth type $2 \mathrm{O}$ disease. Sci Rep 2018;8:1739.

22. Barwick KES, Wright J, Al-Turki S, et al. Defective presynaptic choline transport underlies hereditary motor neuropathy. Am J Hum Genet 2012;91:1103-1107. 
Appendix 1 Author contributions

\begin{tabular}{|c|c|c|c|}
\hline Name & Location & Role & Contribution \\
\hline Nataly I. Montes-Chinea, MD & University of Miami, Miami & Author & $\begin{array}{l}\text { Major role in the acquisition of data design and } \\
\text { conceptualized study; analyzed the data; drafted } \\
\text { the manuscript for intellectual content }\end{array}$ \\
\hline Zhuo Guan, PhD & $\begin{array}{l}\text { Massachusetts Institute of } \\
\text { Technology }\end{array}$ & Author & Major role in the acquisition of data \\
\hline Marcella Coutts, MD & University of Miami, Miami & Author & Major role in the acquisition of data \\
\hline Cecilia Vidal, MD & University of Miami, Miami & Author & Major role in the acquisition of data \\
\hline Steve Courel & University of Miami, Miami & Author & $\begin{array}{l}\text { Major role in the acquisition of data; analyzed } \\
\text { the data }\end{array}$ \\
\hline Adriana P. Rebelo, PhD & University of Miami, Miami & Author & $\begin{array}{l}\text { Major role in the acquisition of data; analyzed } \\
\text { the data; drafted the manuscript for } \\
\text { intellectual content }\end{array}$ \\
\hline Lisa Abreu & University of Miami, Miami & Author & Major role in the acquisition of data \\
\hline Stephan Zuchner & University of Miami, Miami & Author & Drafted the manuscript for intellectual content \\
\hline J.Troy Littleton & $\begin{array}{l}\text { Massachusetts Institute of } \\
\text { Technology }\end{array}$ & Author & $\begin{array}{l}\text { Design and conceptualized study; analyzed the } \\
\text { data; drafted the manuscript for intellectual } \\
\text { content }\end{array}$ \\
\hline $\begin{array}{l}\text { Mario A. Saporta, MD, PhD, } \\
\text { FAAN }\end{array}$ & University of Miami, Miami & $\begin{array}{l}\text { Corresponding } \\
\text { author }\end{array}$ & $\begin{array}{l}\text { Major role in the acquisition of data, design and } \\
\text { conceptualized study; analyzed the data; drafted } \\
\text { the manuscript for intellectual content }\end{array}$ \\
\hline
\end{tabular}




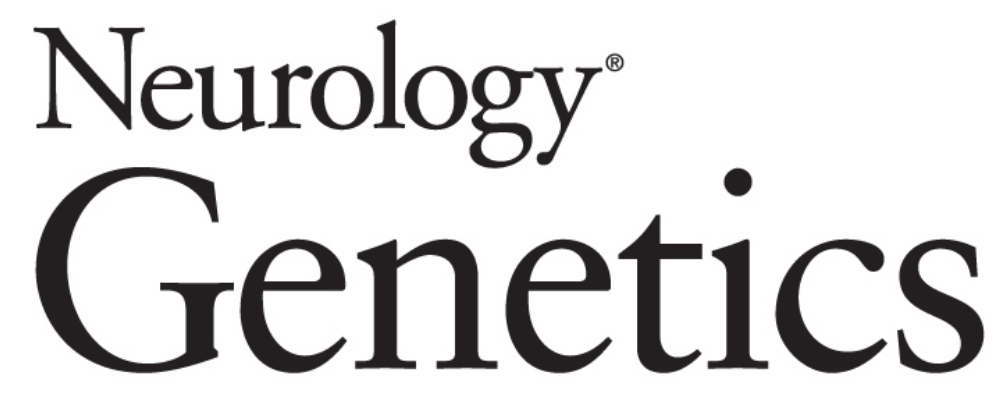

Identification of a new SYT2 variant validates an unusual distal motor neuropathy phenotype

Nataly I. Montes-Chinea, Zhuo Guan, Marcella Coutts, et al.

Neurol Genet 2018;4;

DOI 10.1212/NXG.0000000000000282

This information is current as of October 22, 2018

Neurol Genet is an official journal of the American Academy of Neurology. Published since April 2015, it is an open-access, online-only, continuous publication journal. Copyright Copyright $\odot 2018$ The Author(s).

Published by Wolters Kluwer Health, Inc. on behalf of the American Academy of Neurology.. All rights reserved. Online ISSN: 2376-7839.

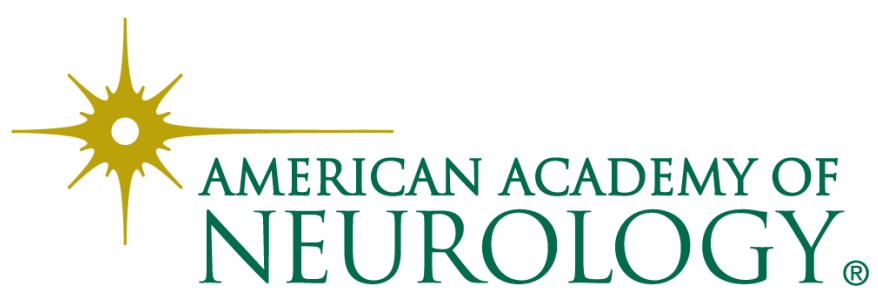




\section{Updated Information \& Services}

References

Citations

Subspecialty Collections

Permissions \& Licensing

Reprints including high resolution figures, can be found at: http://ng.neurology.org/content/4/6/e282.full.html

This article cites 22 articles, 6 of which you can access for free at: http://ng.neurology.org/content/4/6/e282.full.html\#\#ref-list-1

This article has been cited by 2 HighWire-hosted articles: http://ng.neurology.org/content/4/6/e282.full.html\#\#otherarticles

This article, along with others on similar topics, appears in the following collection(s):

\section{All Genetics}

http://ng.neurology.org//cgi/collection/all_genetics EMG

http://ng.neurology.org//cgi/collection/emg

Lambert-Eaton syndrome

http://ng.neurology.org//cgi/collection/lamberteaton_syndrome

Peripheral neuropathy

http://ng.neurology.org//cgi/collection/peripheral_neuropathy

Information about reproducing this article in parts (figures,tables) or in its entirety can be found online at:

http://ng.neurology.org/misc/about.xhtml\#permissions

Information about ordering reprints can be found online: http://ng.neurology.org/misc/addir.xhtml\#reprintsus

Neurol Genet is an official journal of the American Academy of Neurology. Published since April 2015, it is an open-access, online-only, continuous publication journal. Copyright Copyright $\odot 2018$ The Author(s). Published by Wolters Kluwer Health, Inc. on behalf of the American Academy of Neurology.. All rights reserved. Online ISSN: 2376-7839.

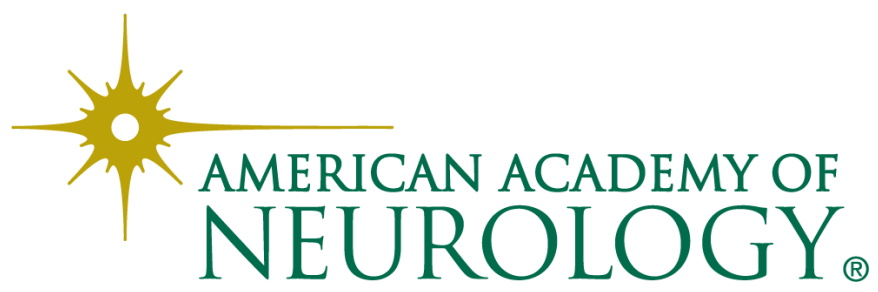

